\title{
La catálisis en la Universidad Veracruzana
}

\author{
María C. Barrera Domínguez, ${ }^{*}$ Carolina Solís Maldonado,** \\ Raúl Alejandro Luna Sánchez,* Benoit Auguste Roger F.,** \\ Sara Núñez Correa***
}

\begin{abstract}
RESUMEN: Este trabajo es una descripción de las actividades de investigación y docencia realizadas en los laboratorios multidisciplinarios de la Universidad Veracruzana (Uv) relacionadas con catálisis y nanomateriales diseñados para este propósito entre otros. En primera instancia se presenta una reseña de la uv desde su creación, así como la misión y visión de esta Institución. Se describen las líneas de investigación, desarrollo y docencia relacionadas con las nanociencias, el trabajo de académicos involucrados con dicho laboratorio en sus diferentes campos de trabajo. Además, se reportan las colaboraciones y proyectos que se llevan a cabo, la infraestructura y los equipos disponibles, así como las actividades de docencia y formación de recursos humanos que se realizan en colaboración con varios cuerpos académicos. Finalmente, se describen los principales logros que se han alcanzado, las áreas de oportunidad y perspectivas de crecimiento que éstos vislumbran a corto y mediano plazo.
\end{abstract}

PALABRAS CLAVE: Líneas de investigación, proyectos de investigación, catálisis, Universidad Veracruzana.

ABSTRACT: In this paper, the research and teaching activities carried out in the different multidisciplinary laboratories at Universidad Veracruzana (uv) that are focused on the fields of catalysis and nanomaterials are described. Firstly, we provide a brief overview of the uv history since its foundation, including its mission and vision. Then, main lines of research, development and teaching, all of them related to nanosciences, are mentioned. Also, the most important works of various researchers investigating in several fields in the aforementioned laboratory are cited. In addition, projects and collaborations with other research groups, available infrastructure and equipment, and teaching and thesis projects carried out by students are included as well. Finally, some of the main achievements of the involved groups, areas of opportunity and perspectives for the future are presented.

KEYWORDS: Research lines, research projects, catalysis, Universidad Veracruzana.

\section{Introducción}

La Universidad Veracruzana inició su existencia formal el 11 de septiembre de 1944. A sus 72 años de creación se ha convertido en la principal institu-

Recibido: 23 de enero de 2017. Aceptado: 26 de abril de 2017.

* Universidad Veracruzana, Facultad de Ciencias Químicas (UV-FCQ), Región CoatzacoalcosMinatitlán, Av. Universidad Veracruzana Km. 7.5 Col. Santa Isabel, C.P. 96535, Coatzacoalcos, Veracruz, Tel.: 01 (921)2115700. Correspondencia: (cobarrera@uv.mx).

** Universidad Veracruzana, Facultad de Ciencias Químicas. Av. Venustiano Carranza S/N, Col. Revolución, C.P. 93390, Poza Rica, Veracruz, México. Tel: 01(782)8227363.

Correspondencia: (casolis@uv.mx).

*** UV-FCQ, Región Coatzacoalcos-Minatitlán. 


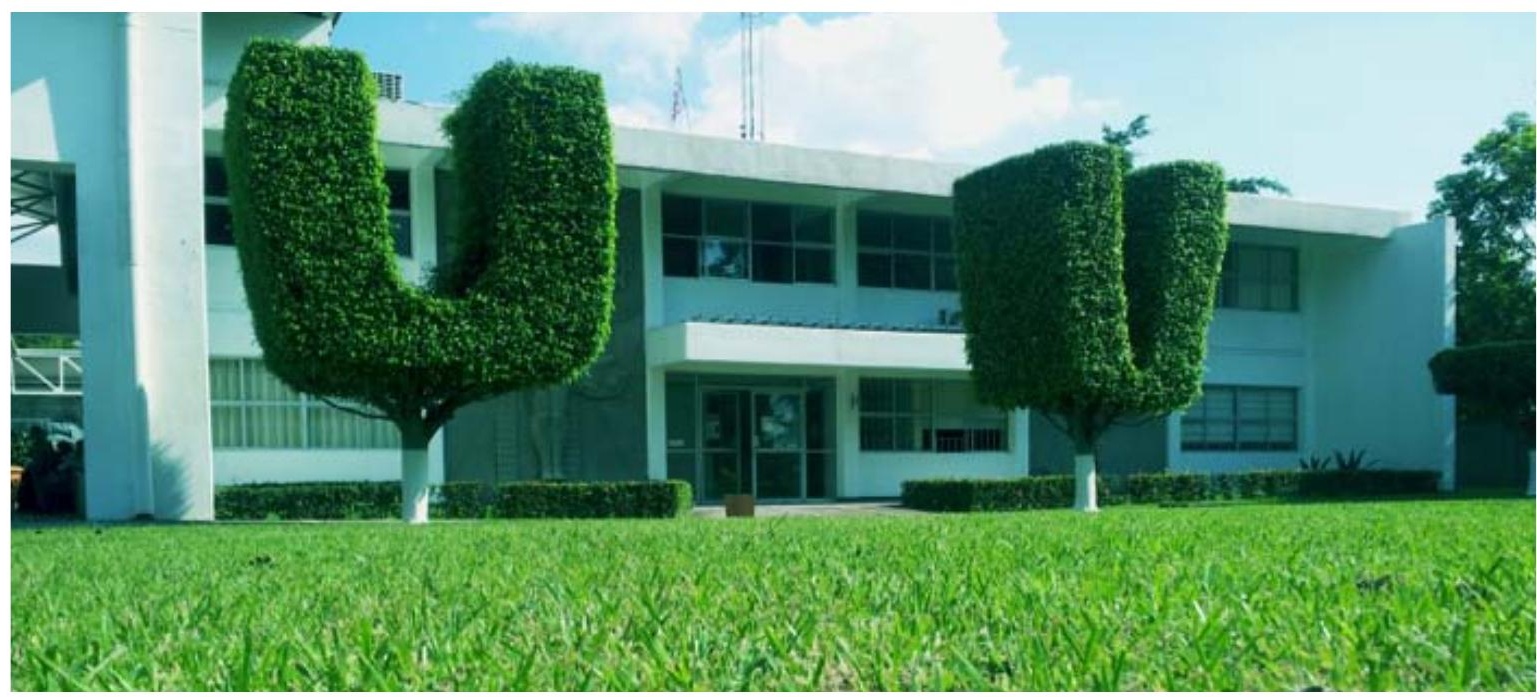

FIGURA 1. Vice Rectoría, Región Poza Rica- Tuxpan.

ción de educación superior pública y autónoma en el estado de Veracruz, con funciones de docencia, investigación, difusión de la cultura y extensión de los servicios.

Los programas de las entidades académicas dedicadas a la investigación se desarrollan en 24 institutos, 18 centros, 2 laboratorios de alta tecnología, el Museo de Antropología y en diversas facultades donde, cada vez más, la investigación se incorpora como el eje de la docencia.

Asimismo, la UV ofrece 74 programas educativos de posgrado, 19 doctorados, 46 maestrías, 3 especialidades médicas, y 6 especializaciones, todas reconocidas por el Padrón Nacional de Posgrados de Calidad (PNPC) del Consejo Nacional de Ciencia y Tecnología (CONACyT). Entre los programas de posgrado, se puede resaltar el doctorado en ingeniería, la maestría en ciencias en micro y nanosistemas, maestría en ingeniería aplicada, maestría en química biorgánica, maestría en gestión ambiental para la sustentabilidad, maestría en ciencias del ambiente, y la especialización en gestión e impacto ambiental [3], que involucra la catálisis como parte de la solución en algunos de los proyectos de investigación desarrollados por parte de los estudiantes. La institución cuenta con 6,025 académicos (22\% doctorado, 37\% maestría, $8 \%$ especialidad y $33 \%$ licenciatura), 33\% son Profesores de Tiempo Completo (PTC). Alrededor del $30 \%$ de los profesores realizan proyectos de investigación mediante diversos programas: servicio social, becas trabajo, becarios del Sistema Nacional de Investigadores (SNI), becarios de distintos proyectos CONACyT, Verano de la Investigación Científica, Verano de la Academia Mexicana de Ciencias y alumnos participantes en otras becas. Esta actividad permite incorporar a los alumnos en los proyectos de investigación con la guía de los 
especialistas de las diferentes áreas, a través de tesis de licenciatura, maestría, doctorado y especialidad.

\section{Cuerpos Académicos y líneas de generación y aplicación de conocimiento}

La investigación en la Universidad Veracruzana, además de desarrollarse en los centros de investigación, también se deriva de los trabajos de tesis de nivel licenciatura y posgrado. Los profesores que realizan actividades de investigación, fortalecen la docencia; asimismo, participan en Cuerpos Académicos (CA) que cultivan diversas líneas de generación y aplicación del conocimiento (LGAC) con impacto a los programas educativos de licenciatura y de posgrado. La UV cuenta con 223 CA que cultivan 393 LGAC; de estos, 10 CA y 14 LGAC realizan trabajos donde la catálisis juega un papel muy importante.

1. CA Fisicoquímica de Procesos: cultiva dos LGAC: a) ingeniería de reacciones químicas, que tiene como actividades: simulación de procesos, formulación y caracterización de materiales catalíticos como con aplicaciones ambientales y energéticas, aplicadas a la remediación ambiental, ahorro energético y producción de combustibles limpios. Fotocatálisis y electrocatálisis. Síntesis de nanomateriales para su uso en la oxidación avanzada aplicando el reactivo Fenton. Modelado de reactores químicos y electroquímicos con la finalidad de conocer el comportamiento de éstos en la síntesis de materiales poliméricos obtenidos por emulsión radicálica viviente. b) Procesos de superficie: encapsulación y liberación de sustancias químicas en la búsqueda de su aplicación en la liberación de fármacos, transporte de genes correlacionando la estructura y propiedades fisicoquímicas.

2. CA Ingeniería, Procesos y Sustentabilidad: cultiva dos LGAC: a) procesos y sustentabilidad: desarrollo y aplicación de tecnologías de vanguardia enfocadas a la prevención y remediación ambiental. Elementos asociados a mejorar de la productividad y competitividad de una industria química más limpia y sustentable que permita coadyuvar con la sociedad a transitar hacia la conservación de la biodiversidad y estilos de vida empáticos con el ambiente. Análisis de la cantidad de energía útil que se puede obtener de un sistema y de los elementos económicos asociados, promoviendo, con lo anterior, procesos con menor demanda de energía para realizar la misma tarea. b) Ingeniería y materiales: síntesis, caracterización y aplicación de materiales avanzados, incluyendo la simulación y modelado, en diversas ramas de la ingeniería. Desarrollo de sistemas catalíticos no agresivos al medio ambiente y capaces de alcanzar altas conversiones hacia productos deseados en el área de la petroquímica. Estudio de sistemas y mecanismos en diferentes condiciones operativas, 
así como el diseño y caracterización de nuevos materiales con aplicación a la prevención y remediación ambiental.

3. CA Ciencias e Ingeniería Aplicada: cultiva tres LGAC: a) catálisis y fotocatálisis: aborda estudios sobre síntesis, caracterización de catalizadores, así como la evaluación de su actividad catalítica. Modelado cinético de los sistemas reaccionantes estudiados, así como efecto de los fenómenos de transporte. b) Tratamientos de aguas $\mathrm{y}$ productos naturales: tratamiento de aguas aplicando procesos avanzados de oxidación. c) Materiales y corrosión: síntesis de materiales poliméricos y biopolímeros. Estudios de corrosión sobre materiales metálicos.

4. CA Ingeniería de los procesos químicos y biológicos: cultiva la LGAC de desarrollo y caracterización de productos que tiene como actividad realizar investigación sobre el desarrollo y caracterización de productos químicos y biológicos a través de estrategias biotecnológicas y/o fisicoquímicas.

5. CA Ingeniería de Procesos y Desarrollo Sustentable: cultiva la LGAC en ingeniería de procesos industriales de separación-reacción y desarrollo de tecnologías limpias, que enfoca sus trabajos a temas como producción de biocombustibles a partir de biomasa.

6. CA Ingeniería, Procesos y Gestión Ambiental: cultiva la LGAC en ingeniería, procesos en el desarrollo sustentable, que tiene como actividad el desarrollar o mejorar los procesos, o atender problemáticas de la región.

7. CA Ingeniería de los procesos químicos y biológicos: cultiva la LGAC en desarrollo y caracterización de productos, que tiene como objetivo correlacionar las características fisicoquímicas con su actividad.

8. CA Química de Productos con Actividad Biológica: cultiva la LGAC en química de productos con actividad biológica.

9. CA Estudio Integral de Ingeniería Aplicada: cultiva la LGAC sobre análisis integral y control de procesos industriales y de ingeniería, en la cual se realiza el trabajo de manera multidisciplinaria.

10. CA Gestión y Control de la Contaminación Ambiental: cultiva la LGAC en bioprocesos de tratamiento de aguas residuales y control de la contaminación de suelos, donde los procesos avanzados de oxidación también tienen un papel importante.

Cabe señalar, que también se colabora en la dirección de tesis de posgrado con otras instituciones de educación superior, como son: la Universidad Autónoma de Nuevo León, Universidad Autónoma de Coahuila, Universidad Autónoma Metropolitana, Universidad Autónoma de Baja California, Universidad Autónoma de San Luis Potosí, e Instituto Tecnológico de Ciudad Madero. 


\section{Infraestructura}

Para lograr el desarrollo de la formación integral de los estudiantes, las facultades de cada región cuentan con infraestructura que les permite realizar proyectos relacionados con la catálisis, se tiene disponible para las labores de investigación los laboratorios multidisciplinarios de investigación, los cuales cuentan con diversos equipos de caracterización fisicoquímica de materiales, además de otros laboratorios de apoyo como son los de ciencia básica.

Entre los equipos para realizar caracterizaciones se pueden resaltar: calorímetro diferencial de barrido, espectrofotómetro UV-vis, espectrofotómetro de espectrómetro infrarrojo, centrifuga refrigerada, analizador termogravimétrico, potenciostato-galvanostato, cromatógrafo de alta resolución de líquidos (HPLC), cromatógrafo de gases (GC), analizador CHN/OS, espectrómetro de absorción atómica, equipo de meteorología, microscopios, balanzas analíticas, autoclaves, viscosímetros, potenciómetros, hornos de convención, centrifuga, rotavapor, así como equipo de cómputo, proyectores y pantallas planas.

Por otro lado, con fin de ofertar estudios de posgrados y crear espacios para el desarrollo de investigación se creó el Instituto de Ingeniería, ubicado en la región de Veracruz, cuyo fin es atender problemas regionales. La investigación que aquí se desarrolla se centra en las áreas de las ingenierías ambiental, de estructuras, eléctrica, hidráulica, mecánica, ciencias de la computación y telecomunicaciones [12].

En esta misma línea, la Universidad Veracruzana tiene la unidad de Servicios de Apoyo en Resolución Analítica (SARA), la cual es una dependencia

FIGURA 2. Equipo de laboratorio, Región Coatzacoalcos-Minatitlán.
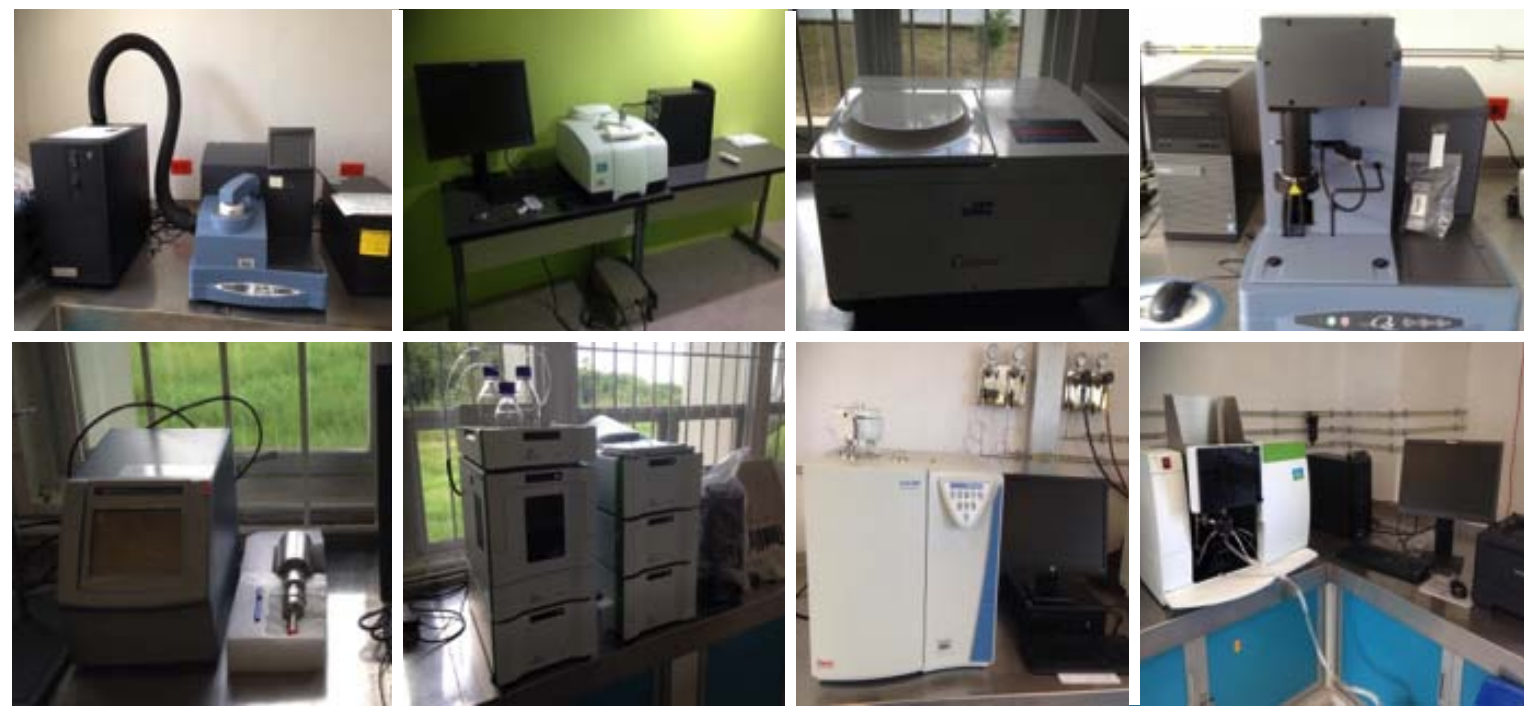
Mundo Nano | CATÁLISIS EN MÉXICo | www.mundonano.unam.mx

10(18), enero-junio 2017 | DOI: 10.22201/ceiich.24485691e.2017.18.60100
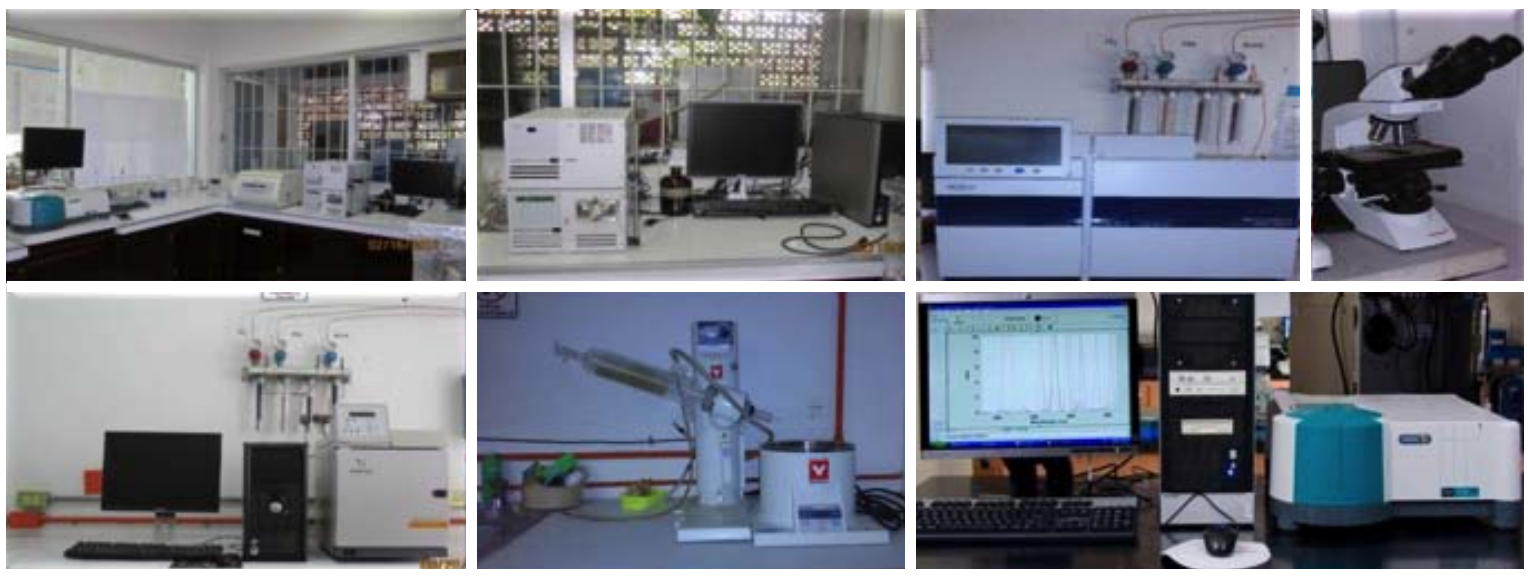

FIGURA 3. Equipo de laboratorio, Región Poza Rica-Tuxpan.

de la Dirección General de Investigaciones y fue creada mediante la participación activa de un grupo de investigadores de la UV, con el fin de ofrecer servicios de alta tecnología a profesionales de la investigación. Los usuarios de la unidad SARA son los investigadores activos adscritos a dependencias intra y extrauniversitarias. La unidad SARA, cuenta con la infraestructura necesaria para desarrollar las técnicas analíticas de resonancia magnética nuclear (RMN), cromatografía líquida de alta resolución (HPLC) y cromatografía de gases acoplada a espectrometría de masas (GC-MS), las cuales son herramientas instrumentales que permiten discernir la naturaleza química de muestras de diversa procedencia. Esto permite una amplia participación en diversas disciplinas además de la catálisis como son biomedicina, química y ciencias ambientales, entre otras [13].

FIGURA 4. Instituto de Ingeniería.
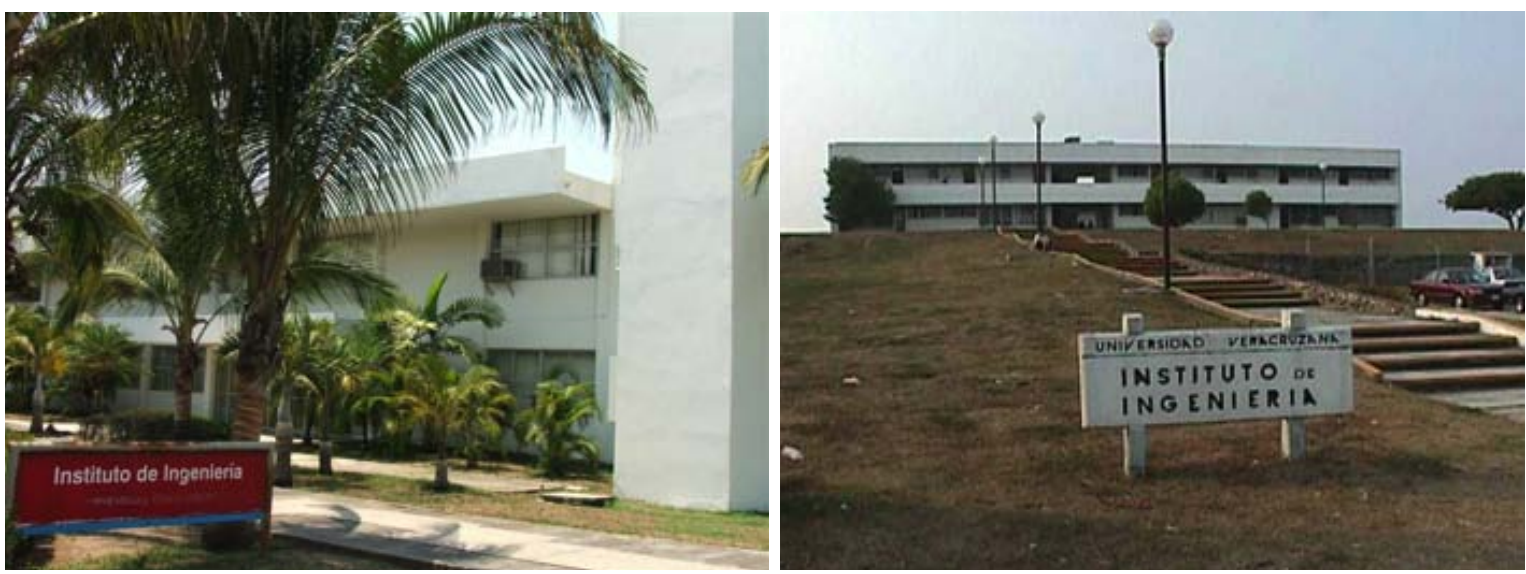
El Centro de Investigación en Micro y Nanotecnología (MICRONA) es una entidad académica de la Universidad Veracruzana, ubicada en la Ciudad de Boca del Río, Veracruz. Previamente inicia sus actividades en el 2003 como un Centro de Diseño en Tecnología MEMs (Sistemas Micro Electro Mecánicos); y en 2005, el Centro de Diseño MEMs expande su portafolio de desarrollo hacia el diseño y caracterización, no sólo de estructuras MEMS, sino de micro y nanotecnología. Uno de los objetivos principales que persigue MICRONA está relacionado con el desarrollo de micro y nanosistemas, en las áreas de instrumentación, sensado y automatización de procesos de manufactura industriales; a través del diseño, fabricación e implementación de micro y nanosistemas. MICRONA contribuye con el desarrollo científico y tecnológico del país, formando recursos humanos de alto nivel en las ramas de la micro y la nanotecnología [14].

En MICRONA, se encuentra el Laboratorio de Materiales Avanzados, donde se lleva a cabo la síntesis y caracterización de nuevos materiales, los cuales generalmente están constituidos por estructuras a nivel micro y nanométrico. Dicho laboratorio cuenta con equipamiento que permite satisfacer la mayoría de las necesidades de investigación a los estudiantes de posgrado durante su trabajo de tesis. El equipamiento se ha obtenido a través de la gestión de equipos para los laboratorios por medio de varios proyectos: propios de la UV, proyectos CONACyT, proyectos de vinculación con la industria. Estos equipos son básicos para el desarrollo de las investigaciones y se encuentran distribuidos, en áreas específicas, de la siguiente manera:

- Fabricación de nuevos materiales: orientada a la fabricación de nuevos materiales, tales como recubrimientos duros, materiales vitrocerámicos, compositos, etc. Para tal efecto, cuenta con un sistema de erosión catódica (sputtering) y un sistema de inmersión (deepcoating), donde los parámetros de crecimiento se encuentran bien controlados.

- Difracción de rayos X: alberga el equipo de difracción de rayos X; se realizan los estudios estructurales de los materiales cristalinos, así como el tipo de fase presente, el tamaño de grano y de manera cualitativa los esfuerzos y deformaciones presentes.

- Síntesis de materiales: corresponde al laboratorio de química, donde se realiza síntesis de diversos materiales mediante técnicas como solgel. Cuenta con lo necesario para realizar dichas síntesis, tales como muflas de altas temperaturas, balanza analítica, parrillas de agitación con temperatura, reactivos necesarios, material de laboratorio, etc.

- Microscopía electrónica de barrido: alberga un microscopio electrónico de barrido de emisión de campo de reciente adquisición; con el cual se puede realizar estudios morfológicos a niveles nanométricos así como obtener la composición química de los materiales fabricados. 
Mundo Nano | CATÁLISIS EN MÉXICo | www.mundonano.unam.mx

10(18), enero-junio 2017 | DOI: 10.22201/ceiich.24485691e.2017.18.60100

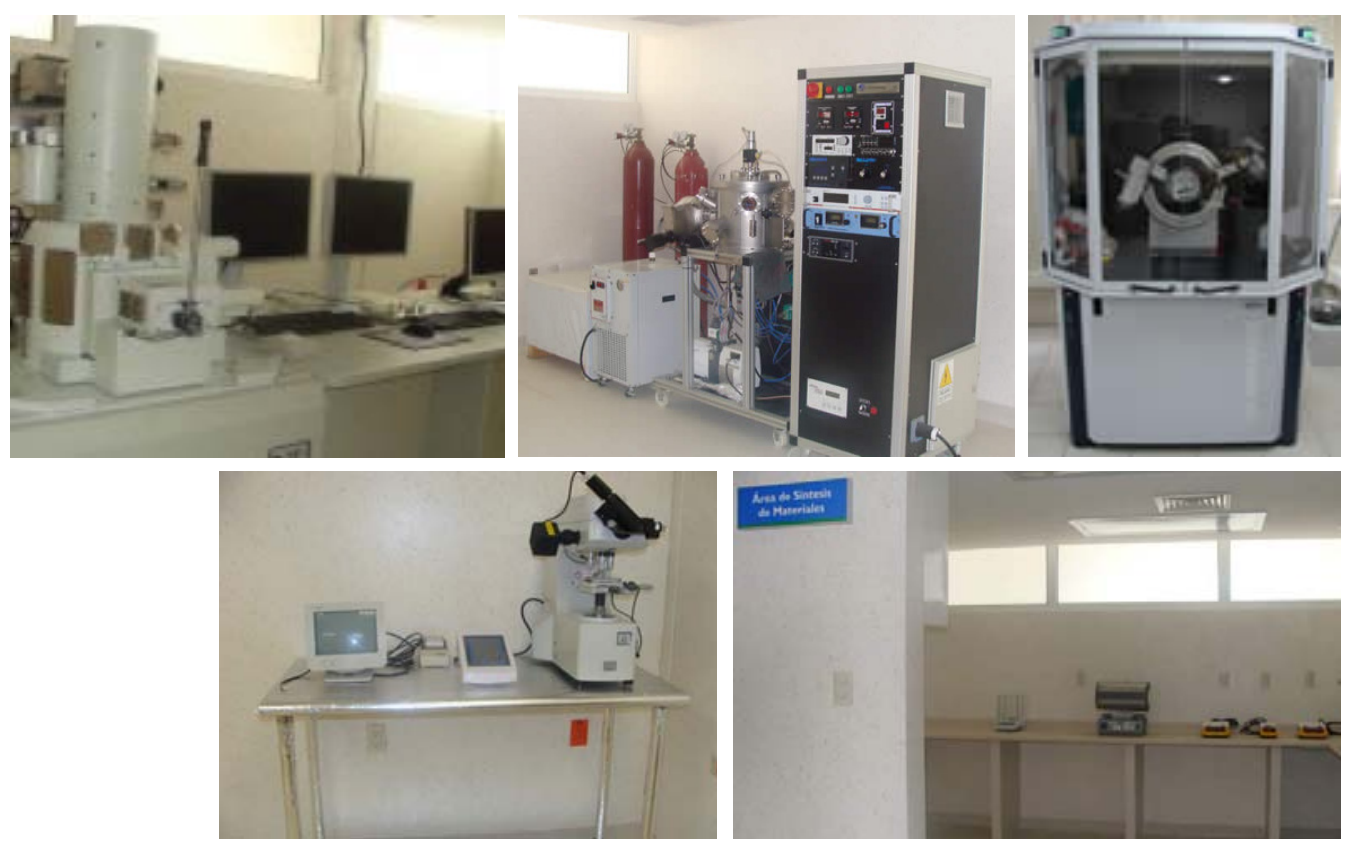

FIGURA 5. Laboratorio de Materiales Avanzados.

- Espectroscopía óptica: aquí se realizan caracterizaciones ópticas de absorción, transmisión, fotoluminiscencia, fotorreflectancia de los materiales fabricados. Cuenta con un láser verde, un monocromador, un amplificador locking, detectores de estado sólido, una fuente de luz (200-1700 nm).

- Dureza y tribología: cuenta con un microdurómetro Vickers y un microtribómetro, equipos destinados para el análisis de propiedades mecánicas de diferentes materiales, así como el comportamiento del desgaste que va sufriendo un material, cuando es sometido a diferentes condiciones de desgaste.

\section{Perspectivas de la catálisis en la uv}

El tema central de este artículo es la actividad en el área de catálisis en la Universidad Veracruzana. El trabajo a futuro es establecer las estrategias a seguir para continuar siendo competitivos a nivel nacional e internacional en los ámbitos de investigación, docencia y vinculación con el sector productivo, entre ellas:

- Formar recursos humanos bien capacitados y dedicados a hacer buena investigación. 
- Mantener un crecimiento razonable en cuanto a publicaciones en revistas internacionales de alto impacto.

- Establecer redes con grupos de investigadores y cuerpos académicos de otras instituciones con miras a fortalecer nuestras líneas de investigación.

- Contar con una visión a futuro para la elección correcta de los campos de trabajo

- Realizar trabajos de investigación tanto en ciencia pura como aplicada.

- Establecer colaboraciones con la industria química mexicana.

\section{Conclusión}

La Universidad Veracruzana cuenta con el personal académico calificado y la infraestructura necesaria para realizar y generar proyectos de investigación en áreas relacionadas con la catálisis. La catálisis es una de las áreas más importantes en la investigación, sobre todo para los programas educativos de ingeniería química, que se imparten en sus cinco campus.

\section{Referencias}

Página institucional:

<www.uv.mx>

Otras:

1. <http://www.uv.mx/percibete/regiones/>

2. <http://www.uv.mx/universidad/doctosofi/informe2015-2016/Discurso-IIIInforme-2015-2016.pdf>

3. 〈http://www.uv.mx/posgrado/oferta-educativa/pnpc/>

4. <http://www.uv.mx/di/general/lineas-de-aplicacion-yo-generacion-delconocimiento/>

5. <http://www.uv.mx/veracruz/mmicrona/lgac/lineas-de-generacion-y-aplicaciondel-conocimiento/>

6. <http://www.uv.mx/veracruz/miaplicada/docentes-e-investigadores/lineasgeneracion-yo-aplicacion-del-conocimiento-del-programa/>

7. 〈http://www.uv.mx/mqb/docentes-e-investigadores/lineas-de-generacion-yaplicacion-del-conocimiento/>

8. <http://www.uv.mx/mgas/docentes/lgac/>

9. <http://www.uv.mx/pozarica/mca/docentes-e-investigadores/lineas-degeneracion-y-aplicacion-del-conocimiento/>

10. <http://www.uv.mx/pozarica/egia/docentes-e-investigadores/lineas-degeneracion-y-aplicacion-del-conocimiento/>

11. <http://www.uv.mx/coatza/cq/> 
Mundo Nano | CATÁLISIS EN MÉXICo | www.mundonano.unam.mx

10(18), enero-junio 2017 | DOI: 10.22201/ceiich.24485691e.2017.18.60100

12. <https://www.uv.mx/veracruz/insting/quienes-somos/historia-mision-yvision/historia/>

13. <https://www.uv.mx/sara/introduccion/presentacion/>

14. <https://www.uv.mx/veracruz/microna/quienes-somos/informacion-general/> 